"This is an Accepted Manuscript of an article published by Taylor \& Francis in International Journal of Mathematical Education in Science and Technology, on-line on 26 Mar 2018 and printed on Volume 49 Issue 8 (pp. 1165-1180), available online: http:/www.tandfonline.com/ DOI: https://doi.org/10.1080/0020739X.2018.1449908"

\title{
Synergy between manipulative and digital artefacts: a teaching experiment on axial symmetry at primary school
}

Eleonora Faggiano, Antonella Montone \& Maria Alessandra Mariotti

This paper presents a study aimed at investigating the didactic potentiality of the combined use of two different kinds of artefacts for the purpose of constructing and conceptualizing mathematical meanings related to the notion of axial symmetry. In our view, the process of meanings construction can be fostered by the use of adequate artefacts but it requires a teaching/learning model which explicitly takes care of the evolution of meanings, from those personal, emerging through the activities, to the mathematical ones, aims of the teaching intervention. The main hypothesis of this study is that a potential synergy may occur between the use of different artefacts, synergy that can foster the integration of different and complementary meanings providing a rich support to the development of the expected mathematical meaning. The Theory of Semiotic Mediation offers the theoretical framework suitable to design the teaching sequence and to analyse the collected data. Specifically, the construct of semiotic potential provides the tool for describing the potentialities of the two artefacts, while that of didactic cycle offers a model for the organization of the different activities. The paper reports on a teaching sequence and its implementation in a teaching experiment, involving pupils at 4 th grade level. We describe them, within the chosen theoretical framework, and provide the analysis of key episodes of the teaching sequence. We show evidence supporting our main hypothesis about the combined use of an artefact that can be manipulated (paper and pin) and a digital artefact (Dynamic Geometry Environment) in the development of the notion of axial symmetry and its properties: the combined, intentional and controlled use of the two artefacts may develop a synergy so that each activity enhances the potential of the other. 
Keywords: Theory of Semiotic Mediation, artefacts, axial symmetry, synergy between artefacts, Primary School

Subject classification codes: $97 \mathrm{C}$

\section{Introduction and rationale}

Generally speaking, in spite of the fact that its importance is widely recognized [1], Geometry has lost its privileged place as a school subject [2,3], and this is true for both its traditional Euclidean approach and its modern transformational approach. As a matter of fact, in Italy, as in other countries, geometric transformations appear in the curriculum, at different school levels, nevertheless, they do not receive much attention by teachers. Geometric transformations also have not been a popular topic in math education research too; a short period of popularity around the seventies of the last century led to studies on the design of teaching sequences at secondary level, like that of Usiskin [4], and successively to some studies focussing on difficulties that students encounter in dealing with certain geometric transformations [5]. More recently the advent of new technologies and specific microworld for Geometry brought this topic to the forefront, mainly at secondary school level [6].

As said, a transformational approach takes on a particularly important role in the field of mathematics. Nevertheless, geometric transformations can become a powerful and effective tool in solving geometrical problems only if they are introduced in a mathematically consistent way: in other terms, if intuitive meanings emerging from actions and observation are suitably formalized into appropriate and well defined mathematical properties. Thus, it becomes crucial for the students to move from a generic perception of regularity to that of correspondence between figures, and subsequently to the idea of transformation (point to point) of the plane in itself. In the 
stream of a previous study [7], we designed a teaching sequence aimed at introducing primary school students to the notion of axial symmetry.

Active student involvement in the use of artefacts is fundamental in mathematics education, and specifically in approaching Geometry at every school level: activities related to the use of artefacts, such as building, drawing, modelling, tracing, measuring and constructing, can develop learners' skills in visualizing and reasoning about geometric relationships [8]. However, to help students discover and focus on the mathematical concepts involved and construct mathematical meanings, the use of artefacts should be well designed and closely monitored. If the use of artefacts is confined to spontaneous activity, indeed, there is no guarantee that students will build bridges from concrete work to the corresponding mathematical concept [9].

Our didactic assumption claims that the process of formalization characterizing a geometrical transformation, such as the axial symmetry, can be achieved through the mediation of specific artefacts. In this paper, we present a teaching sequence, centred on the combined use of two different kinds of artefact, a manipulative and a digital one. The adjective "manipulative" is used with respect to an artefact in the sense that it can be manipulated. Such a meaning is consistent with that reported in other studies, such as in Post [10]. The framework of the Theory of Semiotic Mediation (TSM) [11] provided a suitable reference for the design and the implementation of a teaching sequence: in the following sections we will shortly illustrate the TSM and the two selected artefacts. Then we will focus on the key moments of the teaching sequence where the combination between the two artefacts was assumed to trigger the unfolding of the expected mathematical meanings. The teaching sequence was experimented in a $4^{\text {th }}$ grade class. We will present the analysis of selected episodes drawn from this experiment giving some evidence that the combined, intentional and controlled use of 
manipulative and digital artefacts can develop a synergy whereby each activity enhances the potential of the other.

\section{Theoretical framework}

From a vygotskian perspective, the Theory of Semiotic Mediation (TSM) considers the complex system of semiotic relations between the elements involved in the construction of mathematical meanings through the use of artefacts: the artefact, the task in which the artefact is used, the mathematical knowledge that is the objective of the didactic intervention, and the teaching/learning processes taking place in the classroom.

The notion of artefact here utilized is consistent with the distinction between artefact and instrument, introduced by Rabardel [12] and re-elaborated by Bartolini Bussi and Mariotti [11]: an artefact is any device conceived and realized by human beings for any purpose. The distinction between artefact and instrument aims at distinguishing between the device in itself and the use that any person can be conceived of such a device for a specific purpose. The combination of different schema that might be conceived for its use, with the artefact itself will constitute a new entity, named instrument. The notion of artefact and that of schema of utilization [12] is at the core of the TSM's construct of semiotic potential, that is the twofold relationship that the artefact has with the personal meanings emerging from its use and the mathematical meanings that might be evoked by such use.

[...] on the one hand, personal meanings are related to the use of the artifact, in particular in relation to the aim of accomplishing the task; on the other hand, mathematical meanings may be related to the artifact and its use. This double semiotic relationship will be named the semiotic potential of an artifact. [11, p.754]

The analysis of the semiotic potential will describe what is expected to emerge in the classroom, both actions accomplished and signs produced by the student and its 
relationship with the mathematical meanings that are at stake. For this reason, it is at the core of the design of any teaching sequence and it is the fundamental reference for the analysis of any experimental realization of the didactic intervention. In particular, meanings related to the use of the artefact can be referred to the schema of utilization that are displayed by the students with respect to a specific task. As a consequence, the design of the tasks develops on the base of a fine grain a priori analysis of the solution processes, and specifically on the identification of the schema of utilization that are expected.

According to the TSM, personal meanings emerging from the activities carried out with an artefact may evolve into mathematical meanings that constitute the objective of the teaching intervention. Such an evolution can occur, fostered by specific semiotic activities, in particular, in the peer interaction during the accomplishment of the task and in the collective discussions, orchestrated by the expert guidance of the teacher. The collective construction of shared mathematical meanings is a complex and long process of texture, where it is possible to identify evolution paths (semiotic chains) described by the appearance and enchainment of different types of signs: artefact signs, mathematical signs and pivot signs. The artefact signs are characterized by being highly subjective and clearly related to the specific experience with the artefact, while the mathematical signs constitute the goal of the teaching /learning process and are related to the mathematical meanings as shared in the institution. Pivot signs with their hybrid nature, both referring to the use of the artefact and to the mathematical domain, are characterized by their function in the evolution process, fostering the move from artefact to mathematical signs with their intrinsic ambiguity [11].

Finally, the notion of didactic cycle [11, p.754] constitutes the unit of design: the didactic cycle organizes the coordination between activities with the artefact and 
semiotic activities finalized to make the expected evolution of signs occur. According to this structure, the description of the sequence will be framed by an iteration of didactic cycles.

\section{The artefacts}

As stated above, two different kinds of artefact have been employed: a manipulative and a digital one.

The manipulative artefact consists of a sheet of paper, with a straight line drawn on it marking where to fold it, and a pin to be used to pierce the paper at the right points in order to construct their symmetrical points. This artefact allows an axial symmetry to be created in a direct fashion because the sheet naturally models the plane and the fold allows the production of two symmetrical points using the pin.

The digital artefact has been designed by the Authors to exploit the added value conferred by technology to the use of the chosen manipulative artefact. It is embedded in an Interactive Book (IB) created within the authoring environment of New Cabri (Cabrilog), which allows learning activities to be designed and created, including the objects and tools of a Dynamic Geometry Environment. The Interactive Book appears as a sequence of pages including the designed tasks, together with some specific tools that correspond to specific elements of the manipulative artefact. In particular, among the tools available in the authoring environment, and in agreement with the general principles of dynamic geometry, the tools chosen are: those that allow the construction of some geometric objects (point, straight line, segment, middle point, perpendicular line, intersection point), the "Symmetry" and "Compass" artefacts and the "Trace" tool. A fundamental role is also played by the dragging function, boosted by the "Trace" tool that allows observing the invariance of the properties characterizing the figures. 
The original aspect of this study consists of the choice of two different artefacts and of the didactic assumption that using these two artefacts in an intentionally combined way it is possible to trigger a fruitful synergy between them.

Specifically, it is possible to design a teaching sequence in a way that can connect the semiotic potential of one artefact with the semiotic potential of the other, with the effect that the semiotic potential of an artefact can potentiate the semiotic potential of the other.

\section{Research methodology}

According to the TSM, and specifically according to the main assumption concerning the potential synergy between the two artefacts, a teaching sequence has been designed following the general scheme of successive "didactic cycles"; however, the main hypothesis consists of alternating activities involving the use of one or the other artefact, and of formulating tasks that could exploit the complementarity of their semiotic potentials.

The teaching sequence has been experimented in a first pilot study carried out with the participation of four pairs of pupils attending the fourth year of Primary School. The teaching experiment was developed throughout five lessons, each of them lasting three hours. All the involved pupils were at an average mathematical level. To analyse the teaching experiments, focusing on the unfolding of the semiotic potentials, videotapes and transcriptions were used. Activities were videotaped using two cameras, a fixed one facing the pupils and a second one focused on the artefact in use; conversations were transcribed, which also took into account the specific actions taken with the artefacts.

This paper presents an overview of the whole teaching sequence, made up of six didactic cycles, and a more detailed description of the first two cycles, describing the 
tasks and the semiotic potentials of the involved artefacts. The analysis of some episodes will show not only the unfolding of the semiotic potential related to each of the two artefacts, but also how a synergy between them can foster the construction/conceptualization of axial symmetry and its properties.

\section{Overview of the teaching sequence}

The geometric concept addressed is axial symmetry, in the sense of the isometric transformation of the plane in itself, with a line of fixed points (the axis). From this definition it can be deduced that: axial symmetry preserves the distances and the amplitudes of the angles, and consequently transforms segments into congruent segments and straight lines into straight lines; it is an involutory function [13].

Attention will therefore be paid to those properties of an axial symmetry by means of which it is possible to construct the symmetrical point from a given point with respect to a straight line, in other words the perpendicularity of the axis with respect to the line joining the corresponding points, and the equidistance of the two points from the axis.

As stated above, the design of the teaching sequence follows the general scheme of successive didactic cycles. As regards the design of the activities using the artefact, in accordance with the didactic assumption stated above, we decided that the two types of artefacts might be complementary. We designed didactic cycles where activities involving the use of one or the other artefact were alternated and in order to maintain the connection between them, we formulated tasks that could exploit the complementarity of their semiotic potentials.

In this section, we present an overview of the teaching sequence aimed at developing the mathematical meaning of axial symmetry as a punctual transformation, characterized by specific properties. 
The first didactic cycle involves the manipulative artefact. Through the construction of a symmetric figure with respect to a given line by folding and making punctures with a pin, it aims at firstly introducing the meaning of the punctual correspondence as the correspondence between holes/points. Moreover, asking to compare two symmetric figures of the same figure with respect to two different folding, it aims at firstly introducing a symmetric relation between points and figures depending on the specific folding line.

The second didactic cycle involves the digital artefact. It focuses, once more, on the meaning of symmetry as punctual correspondence, by dragging the given point and observing the resulting movement of the point obtained by means of the button/tool "Symmetry"; and on the key role of the axis by dragging the given line with respect to which the symmetric point has been obtained, and observing the resulting movement of the symmetric point. Moreover, asking to observe what moves and what doesn't move the activities of this second cycle aim at observing the dependence relations through the effects of the dragging as described by the traces. In this second cycle, as shown below, the tasks exploit different aspects of the semiotic potential of the dynamic environment.

The third and forth cycles involve, again alternatively, first the manipulative artefact and then the digital one. The tasks of the third cycle ask to construct the symmetric point of a given point folding along a given line, without the use of the pin. This is possible through the following complex procedure: folding along the given line; folding again along the perpendicular line to the given line which pass through the given point; unfolding the two folds; folding along the perpendicular line; folding once more along the given line; finding the symmetric point by superimposition with the given point. This folding procedure should allow students to focus on two main important properties of axial symmetry: perpendicularity between the axis and the 
segment joining two corresponding points, and equidistance of each of the two corresponding points from the axis.

The forth cycle aims at implementing the same properties in order to construct the symmetric point of a given point, with respect to a given line, without the use of the button/tool "Symmetry". This construction requires the use of the button/tool "perpendicular line" to draw the needed line and the use of the button/tool "Compass" to obtain the symmetric point as the point on the perpendicular line with the same distance from the axis of the given point.

In the fifth and sixth cycles, the tasks to be accomplished are exactly the same but the way in which the two artefacts are used does not correspond. In both cases, it is required to construct the axis that generates a pair of symmetrical points and the properties, previously emerged, need to be employed; however, the way such properties have to be put in relation with the use of each of the artefacts changes. In other words, the properties of the symmetry should drive toward the choice of certain schemes of use, corresponding to specific buttons/tools of the IB and of the specific folding lines of the paper.

In the fifth cycle, the axis can be constructed by drawing the segment joining the two given points and using the "middle point" and the "perpendicular line" buttons/tools. While, in the sixth cycle, the axis can be constructed firstly by folding along the line joining the two given points and then by folding again the paper so that to make the two points overlap.

In the next session we will focus on the first part of the sequence (the first and the second cycle). We will provide a detailed description of the semiotic potential of each artefact related to the given tasks. Then, in the following sections we will present 
some evidence of the unfolding of the semiotic potential as it comes out from the analysis of data collected in the experimentation, and we will discuss these results.

\section{Focus on the first didactic cycle}

In this section we present in detail the first didactic cycle describing the tasks and the semiotic potentials of the artefacts involved.

\section{Description of the tasks}

The first didactic cycle involves three tasks (T1, T2 and T3). Given a figure (convex quadrilateral) drawn (in black) on a sheet, at the moment when handing over the sheet a red line is drawn on it. The task is:

T1. Draw in red the symmetric figure to the black one, with respect to the red line, with the help of the pin:

- fold the sheet along the line

- use the pin to mark the necessary symmetrical points by piercing the paper

- open the sheet and join the holes

After completing this task, on the same paper the teacher draws a blue line. The task is: T2. Draw in blue the symmetrical figure to the black one, with respect to the blue line, with the help of the pin:

- fold the sheet along the line

- use the pin to mark the necessary symmetrical points by piercing the paper

- open the sheet and join the holes.

In the final task, the pupils are asked:

T3. Observe what you have done and write the answers to the following questions: 
- How many times did you point the pin to draw the red figure? Where did you point the pin?

- How many times did you point the pin to draw the blue figure? Where did you point the pin?

- Describe how did you draw the symmetric figure that you get when folding the sheet along a line.

- Look at the red figure and the blue figure. What looks the same about them?

Explain why.

- What looks different? Explain why.

The use of the manipulative artefact, as required by T1, T2 and T3, evokes four important mathematical meanings concerning the notion of axial symmetry: (1.1) the idea of the symmetry axis, expressed by folding the paper along a line; (1.2) the idea of symmetry as correspondence of points, expressed by the holes/points in the paper made by piercing it with a pin; (1.3) the idea of symmetry as a one-to-one correspondence that transforms segments into other congruent segments; as a matter of fact, this property corresponds to the fact that joining the points obtained with the pin yields as product a figure that is the symmetric of the original one; (1.4) the idea that the symmetric figure depends on the axis, expressed by comparing what changed and what didn't change in the two symmetric figures drawn after folding the paper along two different lines.

Taking into account previous results [14] and with the aim of fostering the emergence of specific signs related to the task, some particular expressions, words and phrases, have been chosen purposefully in the formulation:

- To fold along a line, used to refer to the direct action when using the axis; 
- To point, used to refer to the direct action when using the pin to point and pierce to find the symmetric point;

- To join, used to refer to the direct action when drawing a segment between two correspondent points;

- Symmetric figure, used to refer to the direct outcome of the three actions (folding along a line, pointing/piercing on the vertexes of the figure, joining the points/holes);

- What looks different, used to refer to the direct action when comparing two symmetric figures obtained by the same figure with respect to two different lines/axes.

\section{Detailed analysis of the tasks according to the notion of semiotic potential}

When tackling these first three tasks, it can be expected that, the pupils fold the sheet along the line and pierce it using the pin many times on the edge of the figure. In this way we can expect that the meaning of punctual correspondence should emerge, even if the functional dependency can remain implicit. Moreover, the intuitive meaning of line/axis as the element that characterizes the transformation, should emerge from the folding of the sheet, through the superimposition of one figure and the other. Some pupils should foresee that piercing only on the vertexes of the polygonal figure is sufficient. However, pupils could behave in different ways and, for example, decide to use some check strategies, such as labelling the vertexes of the original figure (i.e. A, B, ...) and their holes on the other part of the sheet (i.e. A', B', ...), or making further holes along the sides. Possible doubts concerning the drawing of the resulting figure could also be faced through a continuous visual comparison between points and sides of the given figure and those of the resulting figure. In any case, when these doubts emerge 
they might constitute interesting elements for discussing the problem of the correspondence between segments. Finally, T3 is aimed at making pupils reflect on the invariant aspects and the key role of the axis, when creating a symmetric figure by folding the paper.

According to the TSM and to the specific structure of a didactic cycle, the questions posed in $\mathrm{T} 3$ have been designed and formulated to encourage pupils to describe what they have done in $\mathrm{T} 1$ and $\mathrm{T} 2$. In this way we intend to foster the unfolding of the semiotic potential, so that students' texts contain the expected artefact signs [14].

\section{Focus on the second didactic cycle}

\section{Description of the tasks}

The second cycle involves two tasks (T4 and T5) to be carried out using the digital artefact: specifically, the button/tool "Symmetry" and the dragging function.

Task T4 has been designed as follows. On the first page of the interactive book there are a red line and a point $\mathrm{A}$, while at one side of the page the buttons/tools "Symmetry" and "Name" appear. At the top of the page pupils can read the task: T4. Using the button "Symmetry" construct the symmetrical point of point A with respect to the red line and call it $\mathrm{C}$.

[Clicking on the arrow to continue, the button/tool "Trace" will appear, and then, one at a time at the bottom of the page, the following assignments]

- Activate "Trace" on point A and point C. Drag point A. What moves? What doesn't move? Why? 
- Activate "Trace" on point A and point C. Drag the red line. What moves? What doesn't move? Why?

- Finally, drag point C. What moves? What doesn't move? Why?

According to the notion of "didactic cycle" within the TSM, the T5 aims at fostering the pupils' personal production of signs related to T4. For this reason, the pupils are asked to:

T5. Write down in the summary table below the answers to the questions asked by the interactive book [in T4].

\begin{tabular}{|l|l|l|l|}
\hline Drag & What moves? & What doesn't move? & Why? \\
\hline Point A & & & \\
\hline Red line & & & \\
\hline Point C & & & \\
\hline
\end{tabular}

The use of the digital artefact, required to accomplish T4 and T5, evokes four important mathematical meanings: (2.1) the idea of symmetry as a correspondence of points, expressed by clicking on the button/tool "Symmetry" and then choose a point and a line (or vice versa); (2.2) the idea that the symmetrical point depends on the point of origin, expressed by dragging the point of origin and observing the resulting movement of the symmetrical point; (2.3) the idea that the symmetrical point depends on the symmetry axis, expressed by dragging the axis and observing the resulting movement only of the symmetrical point; (2.4) the idea that the symmetrical point depends on both the point of origin and the symmetry axis, expressed by dragging the symmetrical point and observing the resulting rigid movement of the entire configuration. Moreover, it is important to underline that the effect of the various 
dragging modes is made even more evident by the activation of the "Trace" tool and by the observation of the relations among the trajectories [15].

We emphasize that, as regards the meaning (2.4), in the dynamic geometry environment used, unlike in Cabri Géometrè, for example, it is possible to drag the symmetrical point obtained, and this in fact allows the whole paper to be "shifted".

\section{Detailed analysis of the tasks according to the notion of semiotic potential}

The use of the dragging function in the task T4 allows us to introduce the meanings of co-variation, by means of the dragging action and the observation of the resulting correlated movement of the points. In particular, the request to drag the axis could allow the distinction between independent and dependent variation to emerge. This is related to the different nature of the mutual movements of the points: some points move as a result of the direct action on them that the user attains with the mouse, and other points indirectly move as the result of the movement of those points from which they have been constructed [16].

Moreover, the request to drag the symmetric point, allows the user to underline its specific behaviour with respect to all the other objects of the configuration. When dragging the symmetric point, indeed, it can be observed a resulting rigid movement of the entire configuration. The difference in the movements between the symmetrical point and the point of origin can be compared to the distinction between a dependent variable and an independent variable.

\section{The Hypothesis of synergy between the artefacts}

As stated above, the research hypothesis inspiring the design of the sequence is that when passing from manipulative artefacts to digital artefacts and vice versa, a synergic action will develop in such a way that each activity boosts the learning potential of all 
the others.

In particular, concerning the first part of the teaching sequence, the hypothesis formulated is that the observation the pupils need to make in T4 will cause the concrete experiences they have already had with the manipulative artefact to re-emerge; in other words, we hypothesize that the images on the screen can be interpreted in the light of the previous acts of folding and piercing. In this way we expect that the meanings that have already emerged in relation to the use of the manipulative artefact may be extended, completed and integrated with the specific meanings that should emerge using the digital artefact.

For example, after having constructed the symmetrical point using the specific button, the relation between the two points can be interpreted through the actions of folding and piercing, so the two points can be seen as referring to the two holes. But the meaning of the relation of being symmetrical can be enriched by the distinction between the original point and the corresponding point, thus contributing to the development of the mathematical meaning of a functional - asymmetrical - relation between a point (independent variable) and its symmetrical point (dependent variable).

\section{Analysis of the data}

In this section, according to the theoretical framework of the TMS, we will present some evidence of the unfolding of the semiotic potential through the emergence and evolution of specific signs. Data analysis is based on the transcriptions of students' interactions during the accomplishment of the different tasks, their written answers to the given questions, and finally, the transcripts of the collective discussions concluding each cycle. A specific lens of analysis will regard the identification of key elements supporting our hypothesis of synergy. 


\section{An evidence of signs evolution: from piercing with the pin to the point-to-point correspondence}

With regards to the research hypothesis, in this section we present the analysis of some interesting episodes involving two pairs of pupils (F. \& D., and Au. \& M.). They show not only the unfolding of the semiotic potential and the evolution of signs towards the mathematical meanings, but also the expected synergy between the use of the artefacts in the learning process.

The pupils carried out T1, T2 and T3, and during the following Mathematical Discussion they were asked by the teacher (T.) to describe what they had done, while she would repeat their actions using a sheet of paper and a pin. In the first part, the discussion focused on the choice of the points to be pierced. Not all the pupils had immediately realized that piercing the vertices of the given figure would have been sufficient in order to draw the requested symmetric figure, but shortly all the pupils seemed to have found an agreement on this point. In the following the discussion (see Tab1) concentrated on the use of the pin.

\begin{tabular}{|l|l|}
\hline Transcripts and gestures ${ }^{1}$ & Analysis \\
\hline$(03: 25)$ F.: we must fold the paper along & F. moves her open hand from right to left \\
this red line. So with this pin, after & to simulate the folding, and points to the \\
folding... we must here... see this point?... & red line with her finger. Then F. indicates \\
we must, how can I say? Pierce it with the & the point with her finger, she presses on \\
pin... & it as if she wants to simulate the piercing \\
\hline
\end{tabular}

\footnotetext{
${ }^{1}$ Transcripts and gesture descriptions extracted from the video-recording of the second lesson, concerning the collective discussion at the end of the first cycle.
} 
She takes the pin, shows it, and gives it to the teacher; then she goes toward the paper on the desk and with her finger, presses on the point where the pin should be used to pierce the paper.

(03:33) T. like this? Shall I pierce it?

(Italian translation of the word "Punto?")

She presses the pin on the vertex indicated by $F$. operation.

The act of pointing and pressing her finger on the paper is an artefact sign that mimics the piercing act.

Here the teacher has the paper in front of her, folded along the red line with the black figure facing upward; she asks for confirmation before proceeding, repeating the words used by $\mathrm{F}$. The teacher reflects and accompanies the act of preparing to pierce with the Italian word "punto" accompanied and stressed by the gesture. This word, in Italian, is both a verb and a noun: as a verb it means "pierce", while as a noun it means "point". "Punto" can function as a pivot sign because it evokes the position where to pierce and the piercing itself. In other terms, what the teacher said may evoke the mathematical meaning of point as well as the acting with the artefact, that is piercing. 


\begin{tabular}{|c|c|}
\hline $\begin{array}{l}(03: 36) \text { F. Yeas, but hard, so that it comes } \\
\text { out on the other side }\end{array}$ & $\begin{array}{l}\text { Here clearly emerges the reference to the } \\
\text { idea of a correspondence between the } \\
\text { two points - the origin and the } \\
\text { symmetrical point - coming out of the } \\
\text { action of pointing and piercing with a pin }\end{array}$ \\
\hline $\begin{array}{l}(03: 39) \mathrm{T} \text {. Why must it come out on the } \\
\text { other side? }\end{array}$ & $\begin{array}{l}\text { The teacher invites the pupil to make the } \\
\text { correspondence explicit between the two } \\
\text { holes/points }\end{array}$ \\
\hline $\begin{array}{l}(03: 41) \mathrm{F} . \text { Yes, we need the point to come } \\
\text { out on the other side ...to obtain the figure, } \\
\text { to join the various points and, at the end, } \\
\text { make the figure }\end{array}$ & $\begin{array}{l}\text { The point-to-point correspondence } \\
\text { obtained by piercing the paper with the } \\
\text { pin at the vertices is therefore, in F.'s } \\
\text { view, the thing that makes it possible to } \\
\text { obtain a symmetrical figure. The } \\
\text { meaning of correspondence among } \\
\text { figures has emerged. }\end{array}$ \\
\hline $\begin{array}{l}(03: 58) \text { D. ...that if we do not make the } \\
\text { points... that is, if we do not transfer the } \\
\text { points on the other side, it is almost } \\
\text { impossible to do it [the figure] }\end{array}$ & $\begin{array}{l}\text { The words "transfer" used by D. } \\
\text { reinforce the idea expressed by F. that } \\
\text { the pin must pass through the paper to } \\
\text { the other side. The sign that emerged in } \\
\text { relation to the use of the manipulative } \\
\text { artefact was evolving, thanks to the } \\
\text { shared discussion. In fact, "transfer" can } \\
\text { be considered a pivot sign, because on }\end{array}$ \\
\hline
\end{tabular}




\begin{tabular}{|c|c|}
\hline & $\begin{array}{l}\text { the one hand it may express the action of } \\
\text { piercing through to the other side and on } \\
\text { the other, it refers to the mathematics } \\
\text { sign (transformation) that we aimed to } \\
\text { construct through understanding the } \\
\text { meaning of symmetry as correspondence } \\
\text { between points. }\end{array}$ \\
\hline $\begin{array}{l}\text { (04:33) T. well, so... shall I pierce (Ita. } \\
\text { "punto") all the four vertices? ... and when } \\
\text { I have the four points, what do I have to } \\
\text { do? }\end{array}$ & $\begin{array}{l}\text { After asking all the pupils if they agreed } \\
\text { on the procedure, the teacher pierced the } \\
\text { paper at all the vertices and then } \\
\text { reopened the paper. }\end{array}$ \\
\hline $\begin{array}{l}\text { (10:46) F. we have, firstly, to identify the } \\
\text { points... this one here corresponds to this } \\
\text { one... } \\
\text { She points with the index finger of the right } \\
\text { hand at a vertex of the black figure and } \\
\text { with the one of the left hand at the } \\
\text { corresponding point }\end{array}$ & $\begin{array}{l}\text { The artefact sign "point" has evolved to } \\
\text { the meaning of a point-to-point } \\
\text { correspondence: F. uses the word } \\
\text { "corresponds" }\end{array}$ \\
\hline
\end{tabular}

Table 1. From piercing with the pin to the correspondence of the points This episode shows a first step in the unfolding of the semiotic potential, as expected, but it also illustrates a first evolution towards the mathematical meanings that are the aims of the teaching intervention. The intervention of the teacher (min. 03:33 and 04:33) is fundamental in inducing the pupils to express themselves and so make personal meanings emerge, and the different reformulations showed how such meanings 
evolved from the description of the action to the idea of a correspondence of points, as shown in Fig 1.

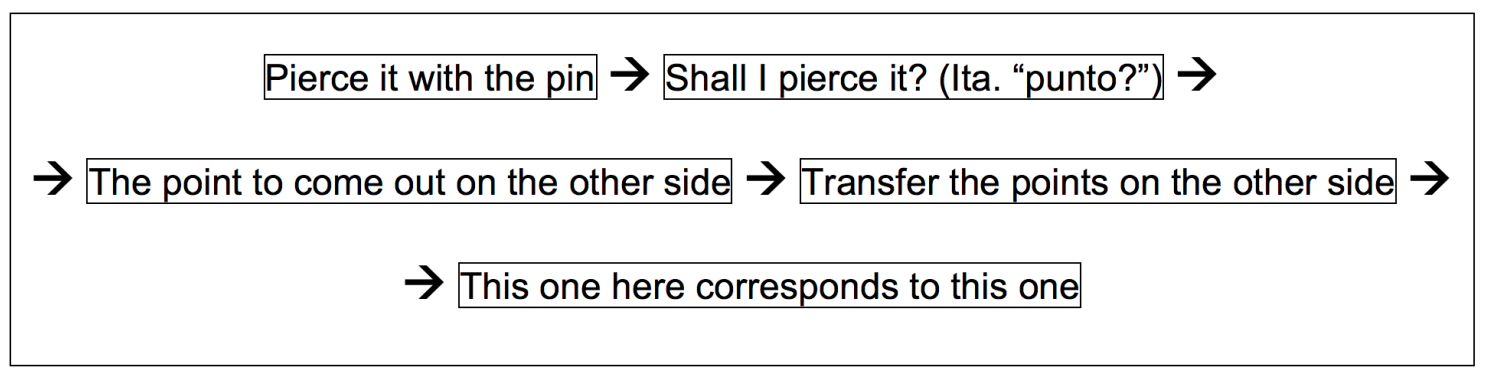

Figure 1. Semiotic Chain: from piercing with the pin to the correspondence of the points

\section{An evidence of the synergy between the artefacts}

This episode occurred during the Mathematical Discussion that was held after concluding T4 and T5. Accomplishing the tasks, the pupils constructed the symmetrical point (C) of a point (A) with respect to a line, using the digital button/tool "Symmetry"; they observed what moved and what didn't move while dragging the point $\mathrm{A}$, the symmetry axis and the symmetrical point $\mathrm{C}$. The analysis of the video recording of the discussion shows two phases of the development of the semiotic mediation process. In the first phase we can observe the unfolding of the semiotic potential of the digital artefact: pupils recognize that point A can be freely moved, but also realize that the symmetric point $\mathrm{C}$ depends on point $\mathrm{A}$ and on the axis, and such dependency becomes even more evident thanks to the effect of the command "Trace". In the second phase, the expected synergy between the use of the two artefacts becomes evident: we can observe how the cross reference to the use of them both, fosters the construction of the mathematical meaning of the functional dependency between points in a symmetry.

Let us analyse the transcript of this second phase of the discussion (Tab 2). Au. is dragging point $\mathrm{C}$ obtained by symmetry and consequently one can observe that both point $\mathrm{A}$ and the symmetry axis is moving. As explained above, dragging $\mathrm{C}$ has the effect of making the digital page/paper shift with the whole set of objects. When the 
teacher asks "What moves? And what doesn't move?" F. answers, perplexed "Everything moves... what doesn't move... nothing at all!". Then she continues to explain her reasoning as in the following excerpt:

\begin{tabular}{|c|c|}
\hline Transcripts and gestures ${ }^{2}$ & Analysis \\
\hline $\begin{array}{l}\text { (13:45) F. when we drag point A, point C } \\
\text { moved but the line didn't!... I can't explain } \\
\text { it... no, but why should it be normal... but } \\
\text { perhaps because C was created by us so... } \\
\text { so in the same way as we did with the } \\
\text { paper... } \\
\text { With her right thumb up she gestures } \\
\text { behind her } \\
\text { (16:14) ... point A is our black figure,... } \\
\text { thanks to the line... since the line moved... } \\
\text { first the red and then the blue... so C } \\
\text { moved. Now... if C moves... everything } \\
\text { moves... why?... }\end{array}$ & $\begin{array}{l}\text { F. has difficulties in understanding why } \\
\text { the two points behave differently when } \\
\text { she drags them. Looking for an } \\
\text { explanation ["why should it be } \\
\text { normal..."], she mentally recalls what } \\
\text { she experienced with the manipulative } \\
\text { artefact and relates the two experiences: } \\
\text { she says "in the same way as we did with } \\
\text { the paper", and associates her words with } \\
\text { a movement of her thumb referring to } \\
\text { what happened before. She associates the } \\
\text { dragging of the line in the interactive } \\
\text { book to the two lines, red and blue, that } \\
\text { were used for the tasks with the paper } \\
\text { and the pin, linking the meanings } \\
\text { emerging during the two experiences. }\end{array}$ \\
\hline
\end{tabular}

\footnotetext{
${ }^{2}$ Transcripts and gesture descriptions extracted from the video-recording of the third lesson, concerning the collective discussion at the end of the second cycle.
} 


\begin{tabular}{|c|c|}
\hline & $\begin{array}{l}\text { counterpart in the work done with the } \\
\text { paper and the pin; then she needs to } \\
\text { reflect further... }\end{array}$ \\
\hline $\begin{array}{l}\text { (17:08) T. hands a sheet of paper to the } \\
\text { pupil } \\
\text { T. let's do this: here is a paper with a line, a } \\
\text { point A, and a pin... }\end{array}$ & $\begin{array}{l}\text { The teacher picks up on her mention of } \\
\text { the tasks on paper and suggests going } \\
\text { further comparing the two experiences... }\end{array}$ \\
\hline $\begin{array}{l}\text { F. takes the paper, folds it and presses on } \\
\text { point A, pierces the paper, removes the pin } \\
\text { and reopens the paper }\end{array}$ & $\begin{array}{l}\text { Using the manipulative artefact } \mathrm{F} \text {. and } \\
\text { starts reconstructing the new situation } \\
\text { proposed by the digital artefact. }\end{array}$ \\
\hline $\begin{array}{l}\text { (17:36) F. now we find the point on the } \\
\text { other side... these are symmetrical... now } \\
\text { let's pretend that A moves here } \\
\text { She folds the paper again, presses the pin } \\
\text { on another point on the paper, pierces and } \\
\text { turns the paper with the pin still inside. } \\
\text { She sees that the pin doesn't come out in } \\
\text { correspondence with the previous hole } \\
\text { (symmetrical to A) but in a different point } \\
\text { and says } \\
\text { (18:03) so C moves. Now... if I move the } \\
\text { line... }\end{array}$ & $\begin{array}{l}\text { She has no difficulty in simulating the } \\
\text { dragging of A, just choosing another } \\
\text { point and pressing the pin on it. She } \\
\text { verifies the effect of this action and notes } \\
\text { the position of the new hole, that is } \\
\text { different from the previous position. } \\
\text { This is expressed by "C moves". } \\
\text { Then in the same way she simulates } \\
\text { dragging the line ("if I move the line"), } \\
\text { making a new fold and pressing once } \\
\text { more on A. She verifies the effect of this } \\
\text { action on the position of the new hole, }\end{array}$ \\
\hline
\end{tabular}




\begin{tabular}{|c|c|}
\hline $\begin{array}{l}\text { She folds again, pierces on } A \text { and turns the } \\
\text { paper } \\
(19: 25) \text { A doesn't move, but only C does... }\end{array}$ & $\begin{array}{l}\text { that is again different from the first } \\
\text { position. } \\
\text { This observation is expressed by "only C } \\
\text { does [move]". }\end{array}$ \\
\hline $\begin{array}{l}\text { (21:49) M. so we can say that if we move } \\
\text { A then } \mathrm{C} \text { will move, if we move the line } \\
\text { then } \mathrm{C} \text { will move...the only one that is } \\
\text { dependent is C... that depends on A and } \\
\text { on the line. }\end{array}$ & $\begin{array}{l}\text { M. offer a synthesis, summarizing what } \\
\text { has been said. The word "dependent" } \\
\text { used by M. reinforces the idea expressed } \\
\text { by F. that dragging free objects produces } \\
\text { a movement on the dependent objects. } \\
\text { The signs that emerged from the synergic } \\
\text { use of the two artefacts are evolving in } \\
\text { the shared discussions. In fact, } \\
\text { "dependent" is a pivot sign that on one } \\
\text { hand expresses the effect of the dragging } \\
\text { action, and on the other the mathematics } \\
\text { meaning of functional dependence. }\end{array}$ \\
\hline (22:22) T. Now, can you move C? & $\begin{array}{l}\text { At this stage the teacher draws F's } \\
\text { attention back to moving the symmetrical } \\
\text { point. }\end{array}$ \\
\hline $\begin{array}{l}F \text {. presses the pin in at point } C \text {, without } \\
\text { folding the paper, points with her finger to } \\
\text { the line she has chosen and then folds the } \\
\text { paper and pierces it at point A }\end{array}$ & $\begin{array}{l}\text { What } F \text {. does at this point is essentially to } \\
\text { reflect on the inverse transformation. She } \\
\text { starts from the symmetrical point and } \\
\text { associates point A to it. She realizes that }\end{array}$ \\
\hline
\end{tabular}


$(23: 20)$ F. ...no because... if I take this C

there will surely be A already on the other side... so if I move C... C must have to move the line otherwise the same point will come out on the other side... oh, no! ... So if it is necessary to move $\mathrm{C}$ it will all have to be moved because it's not possible to move just the symmetrical point! two distinct points cannot be obtained as the symmetrical of the the same point and so the line must necessarily be displaced. It is an indirect argumentation, extremely sophisticated, based on the meaning of the symmetry as a function, on its being univocal and on that each line defines a unique symmetry.

Table 2. Dragging the symmetric point and focusing on the functional dependence This second episode shows how, when using the digital artefact, the meaning of the correspondence between points and that of the axis of symmetry emerge once more. In addition, the excerpt in Tab 2, and in particular the final argumentation expressed by F., concerning the impossibility of a free movement of $\mathrm{C}$, shows how the crossed reference to the use of both the artefacts, combined with the explicit interpretation of one of the experience referring to the other, led to a synergic consolidation of the meaning of functional dependence. A fundamental role is played in the process by the characteristic of this particular digital environment whereby dragging the point of origin and dragging the symmetrical point produces a different behaviour. Unexpected behaviour initially destabilizes the pupils, but then induces them to go back to using the manipulative to find a possible interpretation. The intervention of the teacher is crucial: providing her with the paper, she allows $\mathrm{F}$. to realize the folding and piercing that the pupil had mentally evoked. In this way, F. attempts to interpret the dragging action and its effect, the trace, in terms of the use of the manipulative artefact, so creating a synergy between the two artefacts. The dependence is then linked to the double moving effect of the dragging function, which is typical of the digital artefact, but it is also 
interpreted according to the folding and piercing action which is typical of the manipulative artefact. In summary, the meaning emerges not only through the unfolding of the semiotic potential of the two single artefacts, but rather through the synergy between them, activated by the comparison of the different experiences had using each of them.

\section{Discussion and main results}

In the previous section we presented the analysis of some data carried out according to the theoretical framework of the TMS: we presented some evidence supporting our basic hypothesis, as it emerged during the accomplishment of the different tasks and during the collective discussions concluding each didactic cycle. The analysis of the emergence and evolution of specific signs showed the unfolding of the semiotic potential, and specifically support our hypothesis concerning the synergy between the two artefacts.

The analysis of the excerpt reported in Tab 1, shows the realization of a semiotic chain, from piercing with the pin to the correspondence of the points. Such a chain starts from the artefact signs produced by F., who indicates the point with her finger and presses on it as if she wants to simulate the piercing operation; passes through the teacher's intervention introducing the sign "point" that functions as a pivot sign; and evolves in the sign "point" used by D. to express the correspondence generated by piercing: "(03:58) [...] if we do not make the points... that is, if we do not transfer the points on the other side". The preceding semiotic chain [11] provides evidence of the role of artefacts in triggering the semiotic process. In fact, according to our hypothesis, such a semiotic chain moves from a highly contextualised sign (the word "point"), strictly related to the use of the artefact, to the mathematical sign (the word “corresponds"). 
The analysis of the second episode in Tab 2 shows the development of the complex system of meanings and the texture of their relationship, highlighting the expected synergy between the two artefacts. For instance, after the meaning of correspondence between points had emerged, that of the axis was revisited and developed, interpreting the phenomena observed in the digital environment by using the manipulative artefact: “(13:45) when we drag point A, point $\mathrm{C}$ moved but the line didn't!... [...] so in the same way as we did with the paper...”. Thus the analysis of the excerpt in Tab 2, and in particular the final argumentation expressed by F., concerning the impossibility of a free movement of $\mathrm{C}$, shows how the meaning of symmetry as correspondence between points is indebted to the explicit crossed interpretation of the experience with one of the artefacts by referring to the use of the other. This highlights that, according to our hypothesis concerning the synergy between the artefacts, the use of the digital artefact in the second activity allowed the meaning of symmetry as correspondence between points to re-emerge and to be legitimated.

Thus we can claim that the expected synergy occurred, but also we can state that such a synergy between the two elements of the duo of artefacts was made possible by attaining a process of crossed interpretation, fostered by the sapient intervention of the teacher.

\section{Concluding remarks}

This paper reported on some preliminary results concerning the validity of the hypothesis about the potentialities of using the combination of artefacts as tool of semiotic mediation. The analysis of the data coming from a teaching experiment clearly shows how the potentialities of each single artefact can be exploited and combined for constructing and developing mathematical meanings concerning axial symmetry. 
The key elements that we would like to focus on are the design of the instructional sequence on the one hand, and its implementation on the other hand.

The design of the tasks was guided/inspired by an attentive analysis of the semiotic potential of each of the two artefacts and by the goal of putting the different meanings into relation. The analysis of the collected data shows evidence of how the unfolding of the semiotic potential occurred, but also how the different meanings could be related and integrated to develop expected meanings related to the mathematical notion of axial symmetry. However, it is the attentive and competent intervention of the teacher, able to grasp the opportunity, that contributed to the emergence of the synergy between the two semiotic potential. As a matter of fact, providing F. with the sheet of paper (17:08) was crucial in triggering F.'s interpretation of what was observed on the screen.

We think that, beyond the specific results coming from the analysis presented above, general indications can be drawn concerning the potentialities of combining manipulatives and digital artefacts that might resonate [17]. Specifically, we would like to focus on the role of the teacher and on her specific intervention in the collective discussion: the request of interpreting actions with one artefact in terms of actions with the other seems to be a very effective semiotic process that may emerge either spontaneously or after the teacher's intervention.

The study is still in progress but the results obtained encourage us to go forward, and we expect further confirmation of our hypothesis analysing the data of the whole teaching sequence. Moreover, in accordance with the TSM, and in particular with the didactic cycle model, we intend to verify the emergence of the observed synergy in other sequences of didactic cycles, involving other sets of artefacts and concerning other mathematical concepts. 


\section{References}

[1] Arcavi, A. (2003). The role of visual representations in the learning of mathematics. Educational Studies in Mathematics, 52, 215-241

[2] Jones, K. (2002). Issues in the Teaching and Learning of Geometry. In: Linda Haggarty (Ed), Aspects of Teaching Secondary Mathematics: perspectives on practice. London: RoutledgeFalmer, 121-139

[3] Clements, D. H., and Sarama, J. (2011). Early childhood teacher education: The case of geometry. J. Math. Teach. Educ. 14, 133-148

[4] Usiskin, Z. (1972). The effects of teaching Euclidean geometry via transformations on student attitudes and achievement in tenth-grade geometry. Journal for Research in Mathematics Education, 3, 249-259

[5] Grenier, D. (1990). Construction et étude d'un processus d'enseignement de la symétrie orthogonale: éléments d'analyse du fonctionnement de la théorie des situations, Recherches en Didactique des Mathématiques, 10(1), 5-60

[6] Hollebrands, K. F. (2007). The Role of a Dynamic Software Program for Geometry in the Strategies High School Mathematics Students Employ, Journal for Research in Mathematics Education, Vol. 38, No. 2, 164-192

[7] Jahn, A. P. (1998). Des transformations des figures aux transformations ponctuelles: étude d'une séquence d'enseignement avec Cabrì-Géomètre II. Relation entre aspects géométriques et fonctionnels en classe de Seconde. (Doctoral dissertation)

[8] Kuzniak, A. (2013). Teaching and Learning Geometry and Beyond... Plenary Lecture. In B. Ubuz, C. Haser \& MA. Mariotti (Eds). Proceedings of CERME8 pp. 33-49. Ankara, Turkey: MET University

[9] McNeil, N.M. \& Jarvin, L. (2007). When theories don't add up: Disentangling the manipulatives debate. Theory into Practice, 46(4), 309-316

[10] Post, T. (1981). The Role of Manipulative Materials in the Learning of Mathematical Concepts. In Selected Issues in Mathematics Education. Berkeley, CA: National Society for the Study of Education and National Council of Teachers of Mathematics, McCutchan Publishing Corporation, 109-131

[11] Bartolini Bussi, M. G. and Mariotti M. A. (2008). Semiotic mediation in the mathematics classroom: Artifacts and signs after a Vygotskian perspective, in L. 
English (ed.), Handbook of International Research in Mathematics Education, (second edition), Routledge, 746-783

[12] Rabardel, P. (1995). Les hommes et les technologies; approche cognitive des instruments contemporains. Paris: Armand Colin

[13] Choquet, G. (1964). L'enseignement de la géométrie. Ed. Hermann, Paris.

[14] Mariotti, M.A., Maracci, M. (2012) Resources for the teacher from a semiotic mediation perspective. In G. Gueudet, B. Pepin \& L. Trouche (eds.) From Text to 'Lived' Resources: Mathematics curriculum Materials and Teacher Development. Mathematics Teacher Education, 2012, Volume 7, Part 1, 59-75, Springer. DOI: 10.1007/978-94-007-1966-8_4

[15] Falcade, R., Laborde, C. \& Mariotti, M.A. (2007) Approaching functions: Cabri tools as instruments of semiotic mediation. Educational Studies in Mathematics, Dordrecht: Kluwer, Vol. 66, N. 3, 317 - 333

[16] Mariotti, M. A. (2015). Transforming Images in a DGS: The Semiotic Potential of the Dragging Tool for Introducing the Notion of Conditional Statement. In Rezat, S.; Hattermann, M.; Peter-Koop, A. (Eds.) Transformation-A Fundamental Idea of Mathematics Education. Springer, 156-175

[17] Faggiano E., Montone A., Rossi P.G. (2017). The synergy between Manipulative and Digital Artefacts in a Mathematics Teaching Activity: a co-disciplinary perspective. JE-LKS. Journal of e-Learning and Knowledge Society, vol. 13, 3345, DOI: $10.20368 / 1971-8829 / 1346$ 